\title{
Strong Enhancement of $\varepsilon^{\prime} / \varepsilon$ through Final State Interactions
}

\author{
Elisabetta Pallante* \\ Facultat de Física, Universitat de Barcelona, Diagonal 647, E-08028 Barcelona, Spain \\ Antonio Pich ${ }^{\dagger}$ \\ Departament de Física Teòrica, IFIC, Universitat de València-CSIC, Apartat Correus 2085, E-46071 València, Spain
} (Received 18 November 1999)

\begin{abstract}
We quantify the important effect of strong final state interactions in the weak $K \rightarrow 2 \pi$ amplitudes, using the measured $\pi-\pi$ phase shifts with $J=0$ and $I=0,2$. The final rescattering of the two pions provides a strong enhancement of the $\Delta I=1 / 2$ amplitude, which so far has been neglected in the theoretical predictions of $\varepsilon^{\prime} / \varepsilon$. This correction increases the standard model prediction of $\varepsilon^{\prime} / \varepsilon$ to values in good agreement with the experimental measurements.
\end{abstract}

PACS numbers: 13.25.Es, 11.30.Er, 11.55.Fv, 13.75.Lb

It is well known that, at center-of-mass energies around the kaon mass, the strong $S$-wave $\pi$ - $\pi$ scattering generates a large phase shift difference $\left(\delta_{0}^{0}-\delta_{0}^{2}\right)\left(m_{K}^{2}\right)=45^{\circ} \pm 6^{\circ}$ between the $I=0$ and $I=2$ partial waves [1]. In the usual description of $K \rightarrow 2 \pi$ decays, this effect is explicitly taken into account, through the following decomposition of the relevant isospin amplitudes with $I=0,2$ :

$$
\mathcal{A}_{I} \equiv A\left[K \rightarrow(\pi \pi)_{I}\right] \equiv A_{I} e^{i \delta_{0}^{I}}
$$

It has also been suggested [2-4] that final state interactions (FSI) play an important role in the observed enhancement of the $I=0$ decay amplitude, $A_{0} / A_{2} \approx 22.2$. However, their impact on the direct $C P$-violating parameter $\varepsilon^{\prime} / \varepsilon$ has never been properly estimated.

At lowest order in chiral perturbation theory (ChPT), $O\left(p^{2}\right)$, the decay amplitudes do not contain any strong phase:

$$
\left.\mathcal{A}_{I}\right|_{O\left(p^{2}\right)}=-\frac{G_{F}}{\sqrt{2}} V_{u d} V_{u s}^{*} C_{I} \sqrt{2} f_{\pi}\left(m_{K}^{2}-m_{\pi}^{2}\right)
$$

where $C_{0}=g_{8}+\frac{1}{9} g_{27}$ and $C_{2}=\frac{5}{9} \sqrt{2} g_{27}$, with $g_{8}$ and $g_{27}$ unknown chiral couplings, corresponding to the two lowest-order $\Delta S=1$ operators in the momentum expansion, transforming as $\left(8_{L}, 1_{R}\right)$ and $\left(27_{L}, 1_{R}\right)$, respectively, under the chiral group $[5,6]$. The phenomenological determination of these couplings from $K \rightarrow 2 \pi$, taking the measured phase shifts into account through (1), gives $\left|C_{0}\right| \approx 5.1$ and $\left|g_{27}\right| \approx 0.29$.

The above procedure is not quite consistent, because the strong phases $\delta_{0}^{I}$ are put by hand. Those phases originate in the final rescattering of the two pions and, therefore, are generated by chiral loops which are of higher order in the momentum expansion. Since the strong phases are quite large, especially in the isospin zero case, one should expect large higher-order unitarity corrections. The existing oneloop analyses of $K \rightarrow 2 \pi[4,7,8]$ show, in fact, that pion loop diagrams provide an important enhancement of the $A_{0}$ amplitude, implying a sizable reduction $(\sim 30 \%)$ of the fitted $\left|g_{8}\right|$ value. However, the phase shift $\delta_{0}^{0}$ predicted by the one-loop calculation is still lower than its measured value, which indicates that a further enhancement should be expected at higher orders.

Many attempts have been made to compute the amplitudes $A_{I}$ from first principles [5,6,9-16]. Although those calculations have provided encouraging results, we are still far from getting accurate predictions. In many approaches FSI are not included in the computational framework. This is the case of present lattice calculations [13], which are able to compute only the one pion $\left\langle\pi\left|\mathcal{H}_{\Delta S=1}\right| K\right\rangle$ matrix elements, or estimates at leading order in the $1 / N_{C}$ expansion $[6,10-12]$ (the phases $\delta_{J}^{I}$ are zero at leading order). Other approaches $[10,14,15]$ include some FSI effects, but in a rather incomplete way; however, the fact that the values found for the $A_{0}$ amplitude are larger than in the previous two methods is a clear indication of the important role of FSI.

Unitarity and analyticity constraints permit a resummation of the effects due to FSI [17]. Let us consider the off-shell amplitudes $\mathcal{A}_{I}(s)$, with $s=\left(k_{\pi_{1}}+k_{\pi_{2}}\right)^{2}$ the invariant mass squared of the final two-pion state. In the elastic region Watson final-state theorem relates the imaginary part of the amplitudes $\mathcal{A}_{I}(s)$ to the strong phases $\delta_{l=0}^{I}(s)$ of the partial wave amplitudes $T_{l=0}^{I}(s)$ which describe the rescattering of the two pions in the final state. We can write a once-subtracted dispersion relation for the amplitude $\mathcal{A}_{I}(s)$, which has the following Omnès solution $[2,18]$ :

$$
\begin{aligned}
& \mathcal{A}_{I}(s)=A_{I}^{(0)}\left(s-m_{\pi}^{2}\right) \Omega_{I}(s), \\
& \Omega_{I}(s) \equiv \exp \left\{\frac{\left(s-m_{\pi}^{2}\right)}{\pi} \int_{4 m_{\pi}^{2}}^{\infty} \frac{d z}{\left(z-m_{\pi}^{2}\right)} \frac{\delta_{0}^{I}(z)}{(z-s-i \epsilon)}\right\} .
\end{aligned}
$$

We have imposed the subtraction at $s=m_{\pi}^{2}$, where the amplitude has a zero. The constant $A_{I}^{(0)}$ is the first derivative of $\mathcal{A}_{I}(s)$ at the subtraction point, i.e., the coefficient of the linear term in $\left(s-m_{\pi}^{2}\right)$ of the Taylor expansion around 
$s=m_{\pi}^{2}$; up to small higher-order mass corrections, it is given by the leading $O\left(p^{2}\right)$ term in the chiral expansion.

The dispersion integral in Eq. (3) has an imaginary part and a real part, so that the complete amplitude can be otherwise written as

$$
\mathcal{A}_{I}(s)=A_{I}^{(0)}\left(s-m_{\pi}^{2}\right) e^{i \delta_{0}^{I}(s)} \mathfrak{R}_{I}(s),
$$

where $\mathfrak{R}_{I}(s)$ originates from the real part of the dispersion integral, which is directly related to the strong phase shift. The factor $\Re_{I}(s)$ has never been taken into account in the short-distance calculation of the amplitudes $\mathcal{A}_{I}(s)$.

In this Letter we show how the lacking of such term $\Re_{I}$ does affect numerically both the ratio $A_{0} / A_{2}$ and the direct $C P$ violation parameter $\varepsilon^{\prime} / \varepsilon$. The result that we obtain is the one needed to move both quantities towards the experimental findings.

The dispersion relation in Eq. (3) is strictly valid only in the elastic region. Above the first inelastic threshold a coupled-channel analysis will be demanded [19]. Here we limit ourselves to the very simple numerical analysis in the elastic region, which at $s=m_{K}^{2}$ should give a quite good approximation to the exact result, including inelastic effects.

We have used the simple parametrizations of the strong phases $\delta_{0}^{I}(s)$ given in Ref. [20]. They reproduce well the experimental data up to the $K \bar{K}$ threshold. Other recent parametrizations of the experimental data [21] performed coupled-channel analyses, which we do not need in this simple first step calculation. The $I=2$ amplitude is found to be elastic and well reproduced even up to $1.6 \mathrm{GeV}$. We then evaluated numerically the dispersion integrals up to $1 \mathrm{GeV}$ for $I=0$ and $1.6 \mathrm{GeV}$ for $I=2$.

Taking $s=m_{K}^{2}$, we find that the on-shell $K \rightarrow 2 \pi$ decay amplitudes get the dispersive correction factors:

$$
\mathfrak{R}_{0}=1.41 \pm 0.06, \quad \Re_{2}=0.92 \pm 0.02 .
$$

The central values have been obtained by using the parametrizations of $\delta_{0}^{I}(s)$ quoted as best fits in Ref. [20]. To estimate the errors we have redone the numerical analysis with two other sets of parametrizations, given in Ref. [20], which underestimate and overestimate, respectively, the phase shift data.

Since we have not included inelastic channels, we have not taken into account contributions from energies above $1 \mathrm{GeV}(1.6 \mathrm{GeV}$ for $I=2)$. This is a quite conservative attitude, since those contributions tend to increase (decrease) $\mathfrak{R}_{0}\left(\Re_{2}\right)$ to slightly larger (smaller) values. The details of the numerical analysis will be given elsewhere [19].

The corrections induced by FSI in the moduli of the decay amplitudes $\mathcal{A}_{I}$ have resulted in an additional enhancement of the $\Delta I=1 / 2$ to $\Delta I=3 / 2$ ratio,

$$
\mathfrak{R}_{0} / \Re_{2}=1.53 \pm 0.07 \text {. }
$$

This factor multiplies the enhancement already found at short distances. This is a quite large correction, not taken into account previously, which improves all existing calculations of $A_{I}^{(0)}$. Taking the $\mathfrak{R}_{I}$ correction into account, the experimental $A_{I}$ amplitudes imply the following corrected values for the lowest-order $\Delta S=1$ chiral couplings in (2):

$$
\left|C_{0}\right| \approx 3.6, \quad\left|g_{27}\right| \approx 0.32 .
$$

These "experimental" numbers are not very far from the short-distance estimates obtained in the first of Refs. [6].

It is interesting to compare our results (5) with the ones obtained by using the lowest-order estimate of the strong phases $\delta_{0}^{I}(s)$ in ChPT. We performed one step towards the unitarization of the amplitudes $\mathcal{A}_{I}(s)$ by using $\left(f_{\pi} \sim\right.$ $93 \mathrm{MeV}$ )

$$
\tan \delta_{0}^{0 ; 2}(s)=\sqrt{1-\frac{4 m_{\pi}^{2}}{s}}\left(\frac{2 s-m_{\pi}^{2}}{32 \pi f_{\pi}^{2}} ; \frac{2 m_{\pi}^{2}-s}{32 \pi f_{\pi}^{2}}\right),
$$

which gives the usual representation of $\delta_{0}^{0,2}(s)$ at threshold $\left(s \sim 4 m_{\pi}^{2}\right)$, where $\tan \delta_{0}^{0,2} \sim \delta_{0}^{0,2}$. For $I=0$ the lowestorder ChPT parametrization of Eq. (8) integrated up to $1 \mathrm{GeV}$ gives $\Re_{0}=1.21$, sensitively underestimating the dispersion integral over the fitted phase as expected. For $I=2$ the situation is similar; lowest-order ChPT provides a good description of the strong phase only at low energies. We obtain in this case $\Re_{2}=0.83$, also lower than the value in Eq. (5) obtained using the fitted phase. Note, however, that the ratio $\Re_{0} / \Re_{2}=1.46$, although slightly lower than the value (6), stays within the quoted error bar. This is in agreement with the observation made in Ref. [1] that the difference $\delta_{0}^{0}-\delta_{0}^{2}$ gets smaller chiral corrections than the individual phase shifts.

Implications for $\varepsilon^{\prime} / \varepsilon$. - The most striking consequence of the correction factors $\mathfrak{R}_{0,2}$ is a sizable modification of the numerical short-distance estimates for the direct $C P$-violation parameter $\varepsilon^{\prime} / \varepsilon$. A handy way of writing this quantity, used in all theoretical short-distance calculations up to date, can be as follows [11]:

$$
\frac{\varepsilon^{\prime}}{\varepsilon}=\operatorname{Im} \lambda_{t} e^{i \Phi}\left[P^{(1 / 2)}-P^{(3 / 2)}\right]
$$

where $\lambda_{t}=V_{t s}^{*} V_{t d}$, the phase $\Phi=\Phi_{\varepsilon^{\prime}}-\Phi_{\varepsilon} \simeq 0$, and the quantities $P^{(1 / 2)}$ and $P^{(3 / 2)}$ contain the contributions from the hadronic matrix elements of four-quark operators with $\Delta I=1 / 2$ and $3 / 2$, respectively,

$$
\begin{aligned}
P^{(1 / 2)} & =r \sum_{i} y_{i}(\mu)\left\langle Q_{i}(\mu)\right\rangle_{0}\left(1-\Omega_{\eta+\eta^{\prime}}\right), \\
P^{(3 / 2)} & =\frac{r}{\omega} \sum_{i} y_{i}(\mu)\left\langle Q_{i}(\mu)\right\rangle_{2} .
\end{aligned}
$$

Here, $\left\langle Q_{i}\right\rangle_{I} \equiv\left\langle(\pi \pi)_{I}\left|Q_{i}\right| K\right\rangle, r$ and $\omega$ are given by 


$$
r=\frac{G_{F}}{2|\varepsilon|} \frac{\omega}{\operatorname{Re} A_{0}}, \quad \omega=\frac{\operatorname{Re} A_{2}}{\operatorname{Re} A_{0}},
$$

and the parameter

$$
\Omega_{\eta+\eta^{\prime}}=\frac{1}{\omega} \frac{\left(\operatorname{Im} A_{2}\right)_{\mathrm{IB}}}{\operatorname{Im} A_{0}}
$$

parametrizes isospin breaking corrections. It is usually set to $\Omega_{\eta+\eta^{\prime}} \approx 0.25$ [22] with large uncertainties.

The Wilson coefficient factors $y_{i}(\mu)$ have been computed to next-to-leading order accuracy $[11,13]$. Since the hadronic matrix elements are quite uncertain theoretically, the $C P$-conserving amplitudes $\operatorname{Re} A_{I}$, and thus the factors $r$ and $\omega$, are set to their experimentally determined values; this automatically includes the FSI effect. All the rest in the numerator are theoretically predicted via short-distance calculations, because the leading contributions come from the operators $Q_{6}$ and $Q_{8}$ whose matrix elements cannot be directly measured from the $K \rightarrow 2 \pi$ decay rates.

As a consequence, since the relevant matrix elements $\left\langle Q_{6,8}\right\rangle_{I}$ are usually taken from lattice calculations [13] or large- $N_{C}$ estimates [11], which do not include FSI corrections, this procedure produces a mismatch with the FSI included phenomenologically in the values of $r$ and $\omega$. This can be easily corrected, introducing in the numerator the dispersion factors $\Re_{I}$ that we have estimated. This implies a large enhancement of the predicted value of $\varepsilon^{\prime} / \varepsilon$ by roughly a factor of 2 .

A fast way to estimate the numerical enhancement is through the approximate formula [11]

$$
\frac{\varepsilon^{\prime}}{\varepsilon} \sim\left[B_{6}^{(1 / 2)}\left(1-\Omega_{\eta+\eta^{\prime}}\right)-0.4 B_{8}^{(3 / 2)}\right],
$$

where $B_{6}^{(1 / 2)}$ and $B_{8}^{(3 / 2)}$ parametrize the matrix elements of the QCD penguin operator $Q_{6}$ and the electroweak penguin operator $Q_{8}$, respectively, in units of their vacuum insertion approximation. These parameters are usually taken to be (from Lattice calculations [13] and $1 / N_{C}$ considerations [11]) $B_{6}^{(1 / 2)}=1.0 \pm 0.3$ and $B_{8}^{(3 / 2)}=0.8 \pm 0.2$. Since those estimates do not include the FSI effect, their values should be multiplied by the appropriate factors $\Re_{0}$ and $\Re_{2}$, respectively [23]. However, the term $B_{6}^{(1 / 2)} \Omega_{\eta+\eta^{\prime}}$ in Eq. (13) should be multiplied by $\Re_{2}$ and not by $\Re_{0}$, because it corresponds to two final pions with $I=2$.

The three terms of Eq. (13) should then be corrected to $\left.B_{6}^{(1 / 2)}\right|_{\mathrm{FSI}}=1.4 \pm 0.3,\left.\quad B_{8}^{(3 / 2)}\right|_{\mathrm{FSI}}=0.7 \pm 0.2$, and $\left.B_{6}^{(1 / 2)} \Omega_{\eta+\eta^{\prime}}\right|_{\text {FSI }}=0.23 \pm 0.07$. In the latter, we have set $\Omega_{\eta+\eta^{\prime}}$ equal to 0.25 , disregarding its (probably large) error. This amounts to a FSI enhancement of $\varepsilon^{\prime} / \varepsilon$ by a factor of 2.1.

Thus, the so-called "central" value in Refs. [11], $\varepsilon^{\prime} / \varepsilon=7.0 \times 10^{-4}$, gets increased by the FSI correction to $\varepsilon^{\prime} / \varepsilon=15 \times 10^{-4}$, which compares well with the present experimental world average [24]

$$
\operatorname{Re}\left(\varepsilon^{\prime} / \varepsilon\right)=(21.2 \pm 4.6) \times 10^{-4} .
$$

The calculated FSI corrections provide an all-order resummation of those chiral logarithms generating absorptive contributions to the weak amplitudes. The large effect found in the $I=0$ amplitude is responsible for the bulk of the FSI enhancement of $\varepsilon^{\prime} / \varepsilon$. For $I=2$ the FSI correction is instead small, as expected, and other contributions could be important as well [25].

The final theoretical prediction of $\varepsilon^{\prime} / \varepsilon$ depends on other hadronic and quark mixing parameters, which introduce a rather large uncertainty. Moreover, the usually quoted errors on $B_{6}^{(1 / 2)}$ and $B_{8}^{(3 / 2)}$ are intended to cover only the range of values obtained up to now from lattice and $1 / N_{C}$ calculations; they could be affected by additional systematic uncertainties. It has been said in Refs. [11] and [13] that the theoretical prediction can be made consistent only with the experimental value (14) for rather extreme values of the input parameters, requiring a conspiracy of several inputs in the same direction. The important point we want to stress here is that, once the effect of FSI has been properly taken into account, the experimental value of $\varepsilon^{\prime} / \varepsilon$ can be easily obtained with reasonable values [26] of the different inputs.

Our calculation of the unitarity $\Re_{I}$ correction factors provides a hint on why the Trieste [14] and Dortmund [15] groups were able to obtain larger values of $\varepsilon^{\prime} / \varepsilon$ than the Munich [11] and Rome [13] collaborations. The first two groups did include some FSI corrections, at the one-loop level, which pushed their predictions in the right direction. The use of the dispersive factors $\mathfrak{R}_{I}$ offers a more powerful and consistent way to estimate these FSI effects, because the Omnès exponential $\Omega_{I}(s)$ sums the unitarity logarithms to all orders in the chiral expansion.

More work is still needed in order to get an accurate prediction for $\varepsilon^{\prime} / \varepsilon$. In the meanwhile, our calculation demonstrates that, within the present uncertainties, the standard model theoretical estimates can easily accommodate the experimental value, without any need to invocate a new physics source of $C P$ violation phenomena.

This work has been supported in part by the European Union TMR Network EURODAPHNE (Contract No. ERBFMX-CT98-0169) and by DGESIC (Spain) under Grant No. PB97-1261. E. P. is supported by the Ministerio de Educación y Cultura (Spain).

*Email address: pallante@ecm.ub.es

${ }^{\dagger}$ Email address: Antonio.Pich@uv.es

[1] J. Gasser and U-G. Meissner, Phys. Lett. B 258, 219 (1991).

[2] T. N. Truong, Phys. Lett. B 207, 495 (1988).

[3] N. Isgur et al., Phys. Rev. Lett. 64, 161 (1990).

[4] J. Kambor et al., Nucl. Phys. B346, 17 (1990); Phys. Lett. B 261, 496 (1991); Phys. Rev. Lett. 68, 1818 (1992). 
[5] A. Pich, B. Guberina, and E. de Rafael, Nucl. Phys. B277, 197 (1986).

[6] A. Pich and E. de Rafael, Nucl. Phys. B358, 311 (1991); Phys. Lett. B 374, 186 (1996).

[7] J. Bijnens, E. Pallante, and J. Prades, Nucl. Phys. B521, 305 (1998).

[8] E. Pallante, J. High Energy Phys. 01, 012 (1999).

[9] M. Jamin and A. Pich, Nucl. Phys. B425, 15 (1994).

[10] W. A. Bardeen, A. J. Buras, and J.-M. Gérard, Nucl. Phys. B293, 787 (1987); Phys. Lett. B 211, 343 (1988); 192, 138 (1987); 180, 133 (1986); A. J. Buras and J.-M. Gérard, Nucl. Phys. B264, 371 (1986).

[11] A.J. Buras, hep-ph/9908395; S. Bosch et al., hep-ph/9904408; G. Buchalla et al., Rev. Mod. Phys. 68, 1125 (1996); A.J. Buras et al., Nucl. Phys. B408, 209 (1993); B400, 37 (1993); B400, 75 (1993); B370, 69 (1992).

[12] M. Knecht, S. Peris, and E. de Rafael, Phys. Lett. B 457, 227 (1999); 443, 255 (1998); hep-ph/9910396.

[13] M. Ciuchini et al., hep-ph/9910237; Nucl. Phys. B523, 501 (1998); B415, 403 (1994); Phys. Lett. B 301, 263 (1993).

[14] S. Bertolini, hep-ph/9908268; S. Bertolini et al., hep-ph/ 9802405 [Rev. Mod. Phys. (to be published)]; Nucl. Phys. B449, 197 (1995); B476, 225 (1996); B514, 63 (1998); B514, 93 (1998); V. Antonelli et al., Nucl. Phys. B469, 143 (1996); B469, 181 (1996).

[15] T. Hambye et al., hep-ph/9908232; hep-ph/9906434; Eur. Phys. J. C 10, 271 (1999); Phys. Rev. D 58, 014017 (1998).

[16] J. Bijnens and J. Prades, Nucl. Phys. B444, 523 (1995); J. High Energy Phys. 01, 023 (1999).

[17] F. Guerrero and A. Pich, Phys. Lett. B 412, 382 (1997).

[18] R. Omnès, Nuovo Cimento 8, 316 (1958).

[19] E. Pallante and A. Pich (to be published).

[20] A. Schenk, Nucl. Phys. B363, 97 (1991).
[21] D. V. Bugg, A. V. Sarantsev, and B. S. Zou, Nucl. Phys. B471, 59 (1996); T. Ishida et al., hep-ph/9712230 (Hadron 97); R. Kamiński, L. Leśniak, and B. Loiseau, hep-ph/ 9712337 (Hadron 97); Phys. Lett. B 413, 130 (1997); R. Kamiński, L. Leśniak, and J.-P. Maillet, Phys. Rev. D 50, 3145 (1994); J. A. Oller and E. Oset, Phys. Rev. D 60, 074023 (1999); J. A. Oller, E. Oset, and J. R. Peláez, Phys. Rev. D 59, 074001 (1999).

[22] J.F. Donoghue et al., Phys. Lett. B 179, 361 (1986); A. J. Buras and J.-M. Gérard, Phys. Lett. B 192, 156 (1987); H.-Y. Cheng, Phys. Lett. B 201, 155 (1988); M. Lusignoli, Nucl. Phys. B325, 33 (1989).

[23] The electroweak penguin operator $Q_{8}$ contributes to $K \rightarrow$ $\pi \pi$ at $O\left(p^{0}\right)$ in ChPT. Thus, the $C P$-odd amplitude $\operatorname{Im} A_{2}$ does not vanish at $s=m_{\pi}^{2}$ and the corresponding Omnès solution is modified in a trivial way. One gets the same Omnès exponential $\Omega_{I}(s)$ as in Eq. (3) and the same dispersive factor $\Re_{2}$ as for the $C P$-even amplitude $\operatorname{Re} A_{2}$.

[24] NA31 Collaboration, H. Burkhardt et al., Phys. Lett. B 206, 169 (1988); NA31 Collaboration, G. D. Barr et al., Phys. Lett. B 317, 233 (1993); E731 Collaboration, L. K. Gibbons et al., Phys. Rev. Lett. 70, 1203 (1993); KTeV Collaboration, A. Alavi-Harati et al., Phys. Rev. Lett. 83, 22 (1999); NA48 Collaboration, V. Fanti et al., hep-ex/9909022.

[25] After this paper was submitted for publication, an additional positive one-loop chiral correction to $B_{8}^{(3 / 2)}$ has been obtained by V. Cirigliano and E. Golowich [hep-ph/ 9912513]. On the other side, the $O\left(p^{4}\right)$ isospin-breaking contribution from $\pi^{0}-\eta$ mixing has been calculated by G. Ecker et al. [hep-ph/9912264], who find a smaller value $\Omega_{\eta+\eta^{\prime}}=0.16 \pm 0.03$ and, therefore, a slightly larger central value for $\varepsilon^{\prime} / \varepsilon$.

[26] J. Prades and A. Pich, Phys. Lett. B 346, 342 (1995). 